\title{
Deep Brain Stimulation: Awake and Asleep Options
}

Chengyuan Wu, MD, MSBmE; Ashwini D. Sharan, MD

${ }^{1}$ Department of Neurological Surgery, Thomas Jefferson University, Philadelphia, PA

"Good stereotactic surgery for movement disorders can be performed with or without the microelectrode, and poor surgical results can occur both with and without the microelectrode." - Dr. Roy Bakay

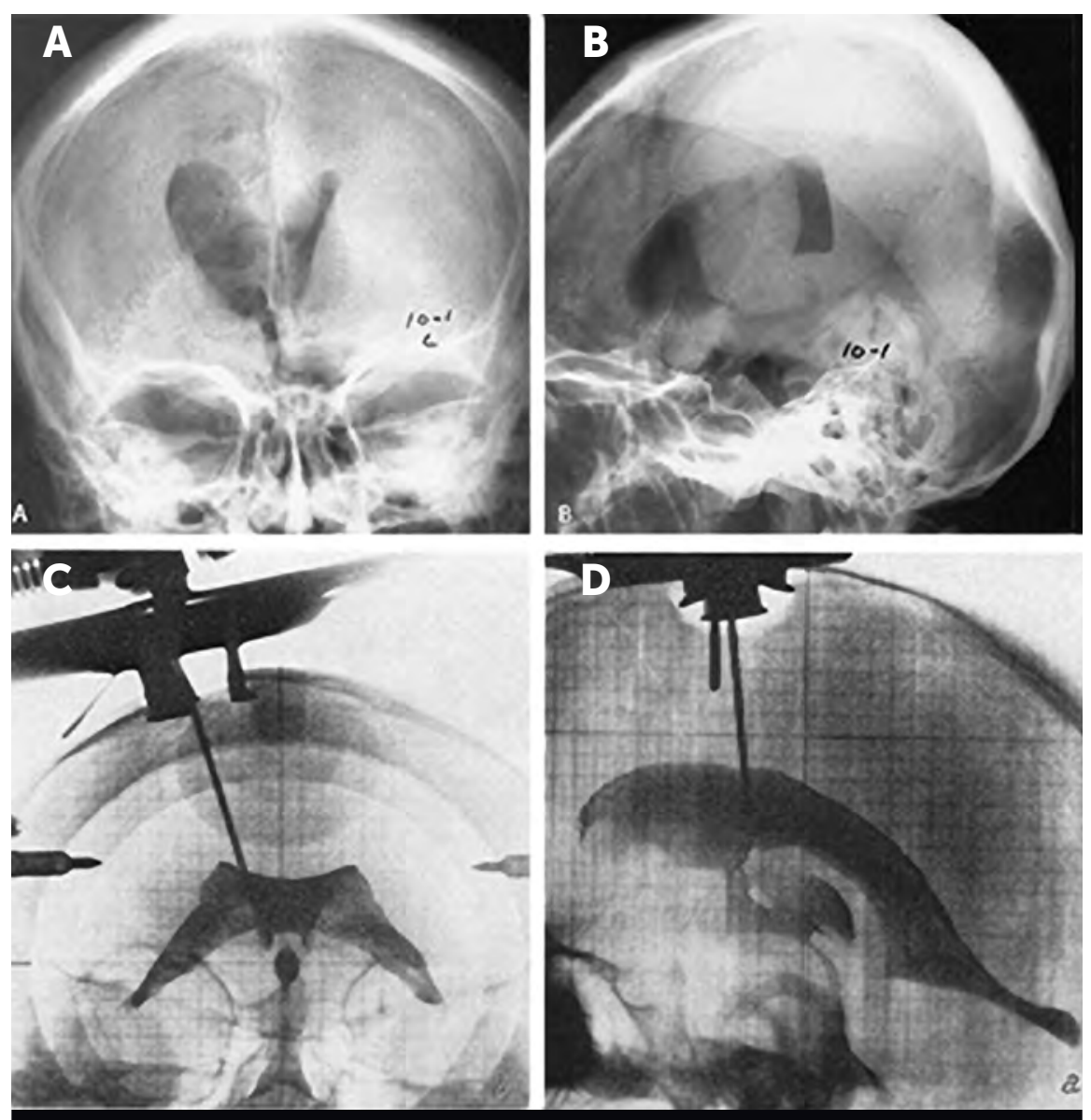

Figure 1

Early methods of localizing deep brain structures. A. Anteroposterior and B. lateral views of a pneumoencephalogram obtained by filling the ventricles with air; and C. Anteroposterior and $\mathbf{D}$. lateral views of a ventriculogram obtained by injecting the ventricles with contrast medium.

\section{A BRIEF HISTORY OF DBS AND NEUROIMAGING}

Stereotactic neurosurgery is founded on the ability to accurately localize and safely access targets within the brain in a minimally-invasive manner. The stereotactic method was first described in 1908 by Sir Victor Horsley and Robert Clarke at University College London, where they developed an apparatus for animal experimentation that allowed them to establish a threedimensional Cartesian coordinate system for targeting. At that time, however, $x$-rays were the only available form of imaging the human body and as such, localizing intracranial targets relied on a combination of knowledge from anatomical atlases and the visualization of a few intracranial landmarks such as the pineal gland or the foramen of Monroe. These landmarks could be visualized by filling the ventricles with air (pneumoencephalogram) or a contrast medium (ventriculogram) [Figure 1]. In 1947, Ernst Spiegel and Henry Wycis created the first human stereotactic frame that allowed for lesioning of deep brain nuclei for the treatment of psychiatric disease. ${ }^{2}$

With imaging limited to $x$-rays alone, a need arose for another means of confirming the appropriate location where a lesion would be made or an electrode would be implanted. Nicholas Wetzel and Ray S. Snider have been accredited with performing the first microelectrode recording (MER) in humans in 1958 during a pallidotomy. ${ }^{3}$ Over time, particularly with the popularization of thalamotomy for the treatment of Parkinson's disease and with a growing appreciation of characteristic recordings of specific nuclei, MER became commonplace in stereotactic neurosurgery.

Over the following decades, deep brain stimulation (DBS) gained favor over ablation due to a lower side effect profile and to the ability to reverse the 
effects of the former. As with early deep brain lesioning, deep electrical stimulation of brain structures was originally introduced as a therapeutic option to treat behavioral disorders or chronic pain. ${ }^{2}$ Natalia Petrovna Bekthereva was the first to implant electrodes into subcortical structures for chronic stimulation for hyperkinetic disorders, but the idea to use chronic stimulation as a therapeutic method did not emerge until Alim-Louis Benabid's report in 1987 on stimulation of the Vim nucleus for treating a patient with tremor. ${ }^{2}$

It is also in this decade that the field of neuroimaging was completely changed by the introduction of magnetic resonance imaging (MRI). Improvements in imaging began a decade earlier, however, when the first clinical computed tomography (CT) scan was performed in 1971 [Figure 2]. ${ }^{4}$ While better than x-rays, CT scans were still limited in their ability to represent soft tissue. This limitation would be addressed by MRI, which also emerged in the 1970s; and the first MR images of a human brain were generated in 1978 [Figure 3a]. ${ }^{5}$ Nevertheless, images generated on clinical MR scanners were still not capable of accurately representing targets such as the subthalamic nucleus (STN) or the globus pallidus pars interna (GPi). As such, DBS targeting continued to rely on previously established locations from accepted atlases, such as that of Schaltenbrand and Wahren. In order to account for anatomical variation between patients, MERs and awake testing were necessary to ensure proper positioning of DBS electrodes.

With sufficient safety and efficacy data, the Food and Drug Administration (FDA) approved DBS as a treatment for essential tremor in 1997 and for Parkinson's disease in 2002. ${ }^{6}$ Now, more than 135,000 patients worldwide have received DBS therapy. ${ }^{7}$ While the majority of centers continue to perform the surgery awake and with MER, there has been a trend towards performing the surgery under general anesthesia and without MER. Improvements in technology and a deeper understanding of MRI physics has allowed for clearer and more accurate representations of intracranial anatomy [Figure $3 b-d]$. We are now able to consistently visualize the borders of deep brain nuclei on MR images acquired on clinical scanners, which in turn facilitates surgical planning and allows for an image-guided, image-verified approach to DBS implantation [Figure 4]. Overall, it is the advances in both surgical technologies and neuroimaging techniques that have allowed for the maturation of stereotactic neurosurgery over the past several years. Here, we will outline both the "awake" and "asleep" versions of the surgical procedure; and provide an overview of the pros and cons of each approach.

\section{DBS VERIFIED BY MICROELECTRODE RECORDINGS AND MACROSTIMULATION}

The most common method of implanting DBS electrodes in the United States involves microelectrode recordings (MERs) and macrostimulation in an awake patient. As mentioned above, this technique allows the surgeon to confirm the target for electrode placement with neurophysiologic recordings and immediate clinical response.

At our institution, the trajectory to the desired target is first planned on a T2 weighted or proton density (PD) MRI as well as on volumetric ( $1 \mathrm{~cm}^{3}$ voxel) T1 weighted gadolinium-enhanced MRI. In order to optimize MERs and the reliability of intraoperative testing, the patient's

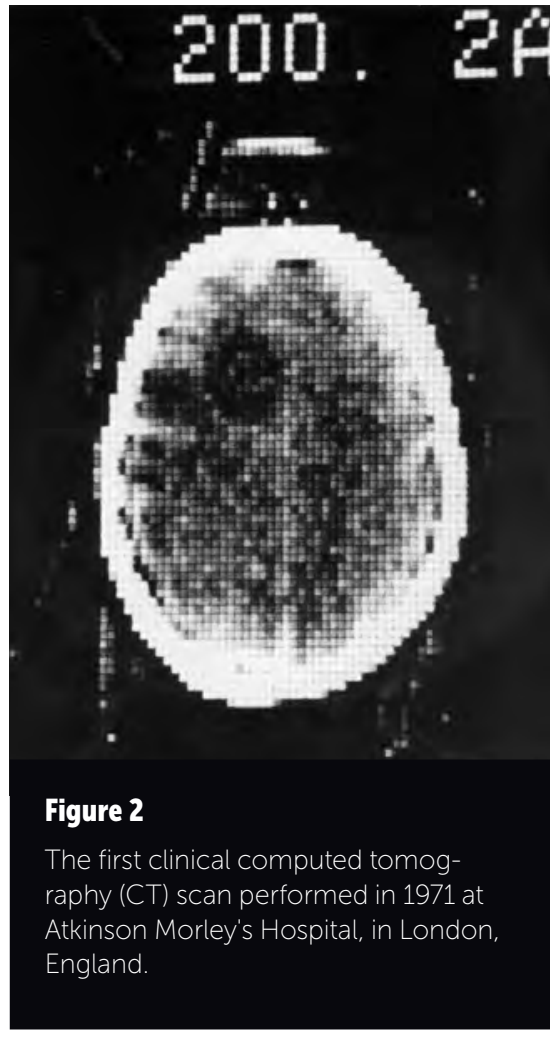

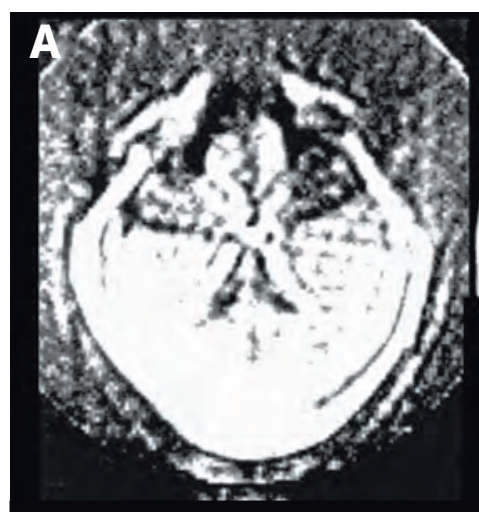
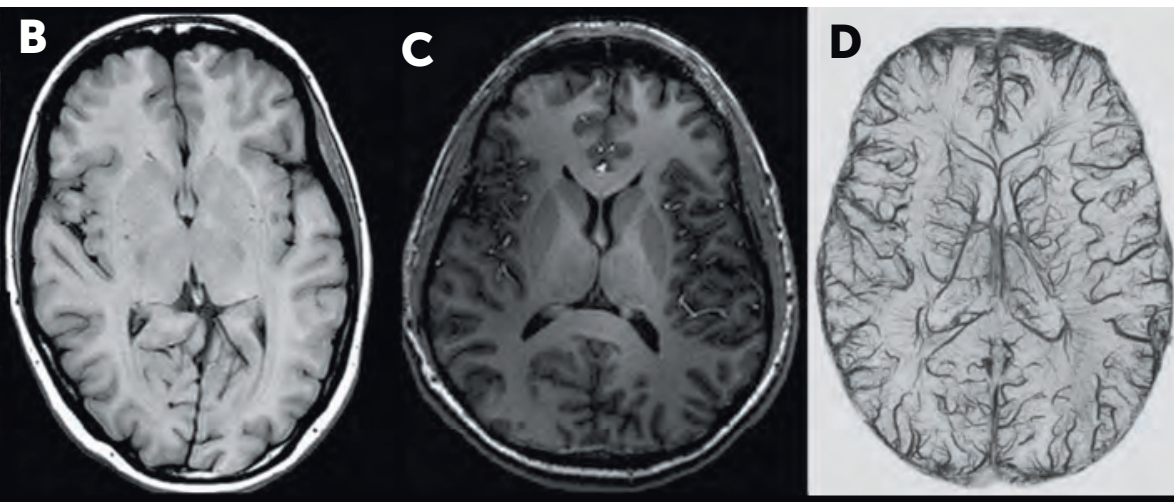

Figure 3

The evolution of magnetic resonance imaging (MRI) A. The first clinical MRI was performed in 1978 in England. Since then, imaging has improved significantly with increasing magnet strength from B. 3T imaging first performed in 1984, C. 7T imaging first performed in 1999, and D. 9.4T imaging first performed in 2007. 

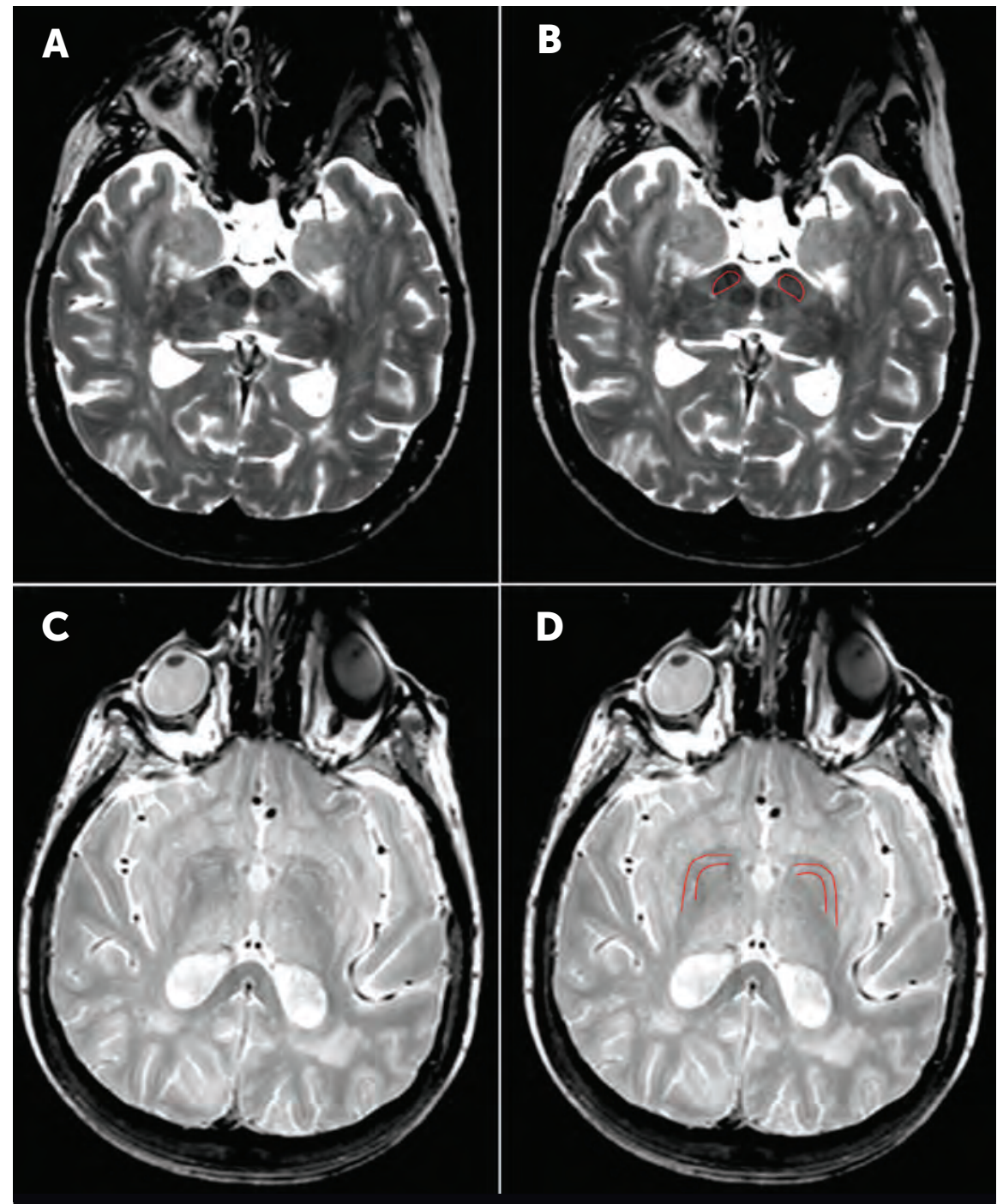

\section{Figure 4}

Magnetic resonance images (MRI) can now be optimized to clearly visualize deep brain structures for targeting in deep brain stimulation (DBS). A. A T2 sequence MRI is used to visualize the subthalamic nucleus (STN) B. highlighted in red; and C. A proton density (PD) sequence MRI is used to visualize the globus pallidus (GP) D. highlighted in red

are in the OFF-state during the time of surgery. The first stage of the procedure starts with placement of a stereotactic (Leksell) frame under local anesthesia. After a reference $\mathrm{CT}$ is acquired with the frame on the patient, this image is merged with the MRI containing the stereotactic plan. This step allows for the calculation of the stereotactic Leksell frame coordinates, which defines the planned room, the patient is positioned in a semirecumbent position with the head fixed to the operating room table. Under monitored anesthetic care, incisions are made in the scalp and $14 \mathrm{~mm}$ burr holes are drilled in the skull. Once the dura and pia have been sharply opened, the microelectrode drive is assembled on the Leksell frame. It is at this time that the patient is awakened and MERs are performed to confirm the desired target based on characteristic neuronal firing patterns [Figure 5]. If MERs do not confirm appropriate placement, a new tract is made to help optimize electrode position. The location of this tract is typically offset by $2 \mathrm{~mm}$ from the original tract and depends on the MERs and the neurosurgeon's knowledge of the surrounding anatomy. Only after MERs have been optimized and the most suitable location has been mapped is the electrode implanted. Across multiple centers, the average number of MER tracts has been reported to be 2.3 for each implanted electrode.8,9 After the DBS electrode has been placed into the desired deep brain target, it is subjected to intraoperative test stimulation in order to confirm therapeutic efficacy and ensure that there are no associated side effects. If a second electrode is to be implanted, this process is repeated. With both electrodes in their final location, the ends of the wires are capped and tunneled under the skin to a point behind the patient's ear. In a review of the literature, the mean operating room time for a unilateral DBS implantation was reported to be 223.83 minutes: and 279.79 minutes for simultaneous bilateral implantation. ${ }^{10}$

The second stage of the procedure consists of connecting the intracranial electrode wires to an implantable pulse generator. This portion of the procedure is always performed under general anesthesia, and may be performed either on the same day as the first stage, or in a delayed fashion as an outpatient procedure.

\section{DBS VERIFIED BY INTRAOPERATIVE IMAGING}

Over the past several years, a growing number of centers have started to offer DBS surgery under general anesthesia or "asleep" DBS. The main premise of this approach is that MRI technology is now capable of clearly identifying the anatomy of the deep brain target; and that intraoperative imaging can be used to verify the location of the DBS lead in this target. As such, both $\mathrm{MR}^{11-13}$ and $\mathrm{CT}^{14-17}$ have been used to accomplish the necessary task of image verification in "asleep" DBS. Although intraoperative MRI allows for direct visualization of the electrode 


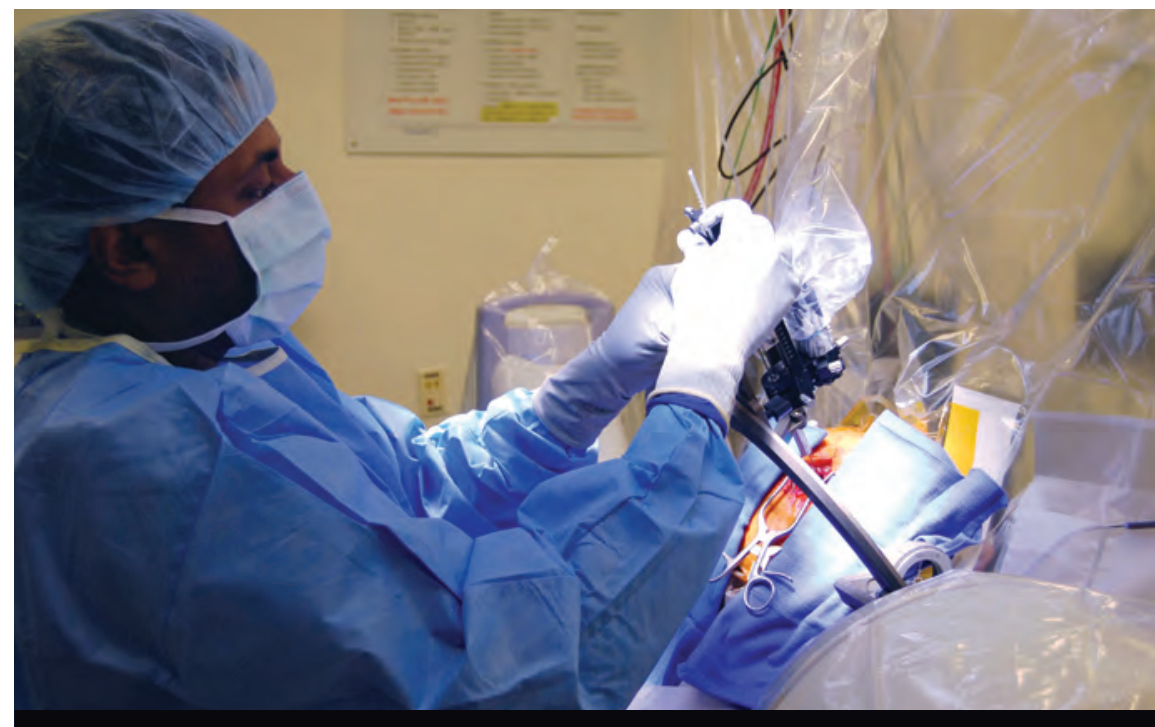

\section{Figure 5}

Dr. Ashwini D. Sharan inserts the DBS electrode into its final position after verifying its optimal position with microelectrode recordings. Macrostimulation will then be used to test for therapeutic effect and rule out side effects from stimulation.

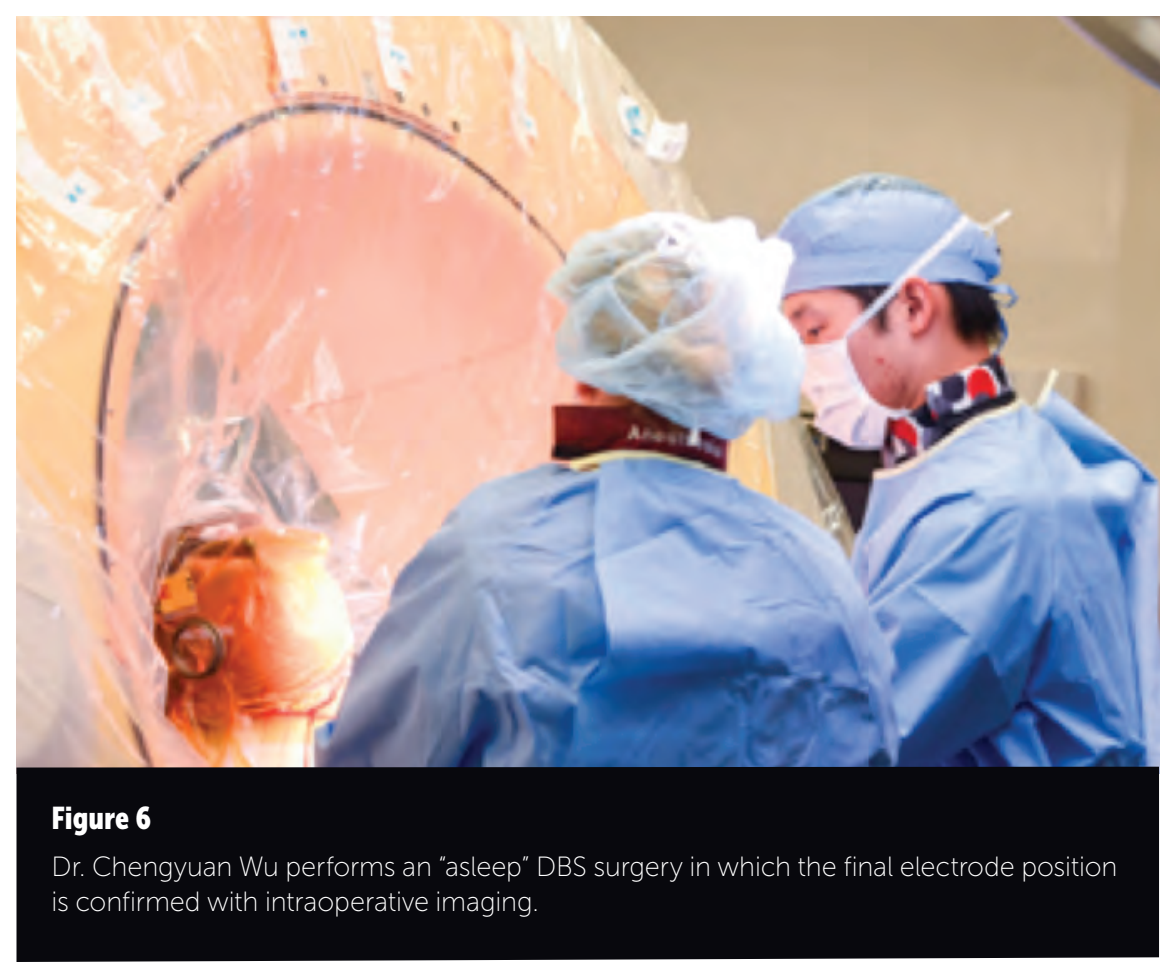

without the need for image fusion, the use of intraoperative CT has been found to be safe and accurate as well. ${ }^{18}$

At our institution, the trajectory to the desired target is once again planned placement of a stereotactic (Leksell) frame under local anesthesia. After a reference $C T$ is acquired with the frame on the patient, this image is merged with the MRI containing the stereotactic plan. Again, this step allows for the calculation of the stereotactic Leksell frame coordinates or robotic (Renishaw Neuromate) arm position, which defines the planned trajectory in real space. In the operating room, the patient is placed under general anesthesia and positioned in a supine position with the head fixed to the operating room table or to the stereotactic robot. The intraoperative CT (Medtronic O-Arm) is also brought into position around the surgical field. Incisions are made in the scalp and $2 \mathrm{~mm}$ twist drill holes are drilled into the skull [Figure 6]. After the dura has been opened sharply and with cauterization, a radiofrequency probe is passed down the planned trajectory in order to measure impedances, which helps to confirm a target in gray matter. The electrode is implanted at this location and secured in place to the skull. The same process is repeated for the second electrode before an intraoperative CT scan is acquired. Before closing the incisions, this intraoperative image is merged back to the original plans and the accuracy of electrode placement is critically assessed. Only if the electrode is within the intended deep brain nucleus and within $2 \mathrm{~mm}$ of its intended trajectory is it considered to be in an appropriate position. This verification step is critical and systematic analysis of targeting errors permits development of strategies to improve surgical accuracy and precision during subsequent procedures. ${ }^{19}$

With both electrodes in their final location, the wires are tunneled under the skin to a point behind the patient's ear. The second stage of the procedure consists of connecting the intracranial electrode wires to an implantable pulse generator, which is usually performed on the same day.

\section{EQUIVALENCE BETWEEN TECHNIQUES}

On a T2 weighted or proton density (PD) MRI as well as on volumetric $\left(1 \mathrm{~cm}^{3}\right.$ voxel) T1 weighted gadoliniumenhanced MRI. The first stage of the procedure similarly starts with

\section{Clinical Outcomes}

In a large multicenter study performed in the UK and published in 2010, 366 
patients with Parkinson's disease across 13 centers were randomized to surgery and best medical therapy or to best medical therapy alone. They found that there was a significant improvement in quality of life ratings, specifically with mobility, activities of daily living, and bodily discomfort in patients that received surgical intervention in addition to medical therapy. ${ }^{20}$ Specifically, patients who underwent MER-guided DBS for Parkinson's disease have demonstrated a 26-33\% improvement in UPDRS-III motor scores after 6 months. ${ }^{12,21,22}$ Furthermore, these effects are long lasting with substantial benefits for symptoms such as tremor, rigidity, and motor complications; whereas other symptoms of akinesia, axial signs, and cognition often continue to deteriorate as part of the natural progression of the disease. ${ }^{23-25}$

The outcomes in patients undergoing "asleep" DBS has certainly been comparable, with patients experiencing a $40-66 \%$ improvement in UPDRS-1II motor scores after 6 months. ${ }^{15,26,27}$ Similarly, 41 patients who underwent "asleep" STN DBS continued to demonstrate significant reduction of motor fluctuations, dyskinesias, and demands in dopaminergic medications at 5 years after implantation; however axial symptoms and bradykinesia continued to worsen as part of disease progression. ${ }^{28}$ A direct comparison between the two approaches was performed by Saleh et al, who performed a retrospective review in which they compared 14 patients who underwent DBS placement under general anesthesia to 23 patients who underwent DBS placement while awake with MER. After 6 months of therapy, both groups showed statistically similar reductions in levodopa equivalent dosages. ${ }^{29}$

\section{Associated Costs}

A 2011 review of literature to date suggested that MER doubled, or even tripled, the cost of DBS implantation compared to surgery performed without MER. ${ }^{10}$ Given its retrospective nature, however, this study compared costs across multiple centers and was limited to the reported costs associated with the surgery itself. More recently, a single center study from Oregon Health and Science University reported no significant difference in cost between "awake" $(\$ 40,052 \pm \$ 6,604)$ and "asleep" (\$38,850 $\pm \$ 4,830)$ DBS when they compared 53 "awake" to 158 "asleep" procedures performed over a 5 year span at their institution. ${ }^{30}$ While they reported a lower variation in procedural costs, it is important to note that this study also include all costs incurred both 30 days before and after surgery.

\section{BENEFITS OF "AWAKE" DBS}

Use of MER in an awake patient for DBS implantation certainly has a tried and true track record. It can be performed reliably even when the borders of intended deep brain target cannot be directly visualized. While clinical MR scanners are generally capable of imaging many of these targets, expertise in both MR physics and the surgical technique of DBS are necessary to establish protocols for the necessary image sequences. Furthermore, not all clinical targets are yet visible on clinical MR scanners-the borders of the ventral intermediate nucleus (Vim) of the thalamus still cannot be clearly distinguished. In such scenarios, the use of MERs increases the accuracy of DBS electrode placement. ${ }^{31,32}$ Even when the borders of the target, such as the subthalamic nucleus (STN) or globus pallidus pars interna (GPi), can be clearly seen, some have pointed out that the subregion of the sensorimotor region of this nucleus cannot be visualized and discerned from the cognitive and limbic regions of the nucleus. ${ }^{33}$

Another major benefit of "awake" DBS is the ability to perform intraoperative stimulation testing. The ability to immediately confirm the therapeutic efficacy of stimulation as well as ensure the absence of no side effects can certainly be reassuring not only for the neurosurgeon, but also for the patient undergoing the procedure. Finally, by having the procedure performed awake, the risks of undergoing general anesthesia are completely eliminated. It is for these reasons that many centers continue to perform DBS in this manner.

\section{BENEFITS OF "ASLEEP" DBS}

\section{Increased Patient Comfort}

While intraoperative testing provides immediate feedback, the time requirement of both MERs and stimulation testing for which the patient must be awake can be challenging for patients, who can fatigue, lose concentration, and in doing so, potentially reduce the reliability of this intraoperative testing. ${ }^{34}$ In contrast, "asleep" DBS is associated with increased patient comfort, reduced anxiety, less back pain, and fewer anesthetic concerns about respiratory difficulties. ${ }^{19,35,36}$ In addition, since no intraoperative neurophysiologic or clinical testing is being performed there is no need to force the patient to an OFF-state; and the lack of complete levodopa reduces tremor severity, painful "off" dystonia or rigidity, "off" anxiety, and confusion for the patient. ${ }^{19,27,35,36}$ The result of all of these factors is more rapid mobilization after surgery and a overall shorter recovery period. Lastly, although more difficult to interpret than in awake patients, MERs can even be performed during "asleep" surgery and has been reported by a few institutions. ${ }^{34-36}$

\section{Shorter Operating Time}

In eliminating the need for intraoperative recording and testing, "asleep" DBS also tends to offer shorter surgical times. As greater comfort and experience is gained with this technique, operative times have improved. ${ }^{29}$ In a retrospective analysis, surgical time was reduced by an average of 175 minutes if a single electrode was implanted without MER. ${ }^{10}$ Such a reduction of the duration which a patient must remain on an operating room table has been suggested to decrease the risk of venous thrombosis and pulmonary embolism. ${ }^{34}$ In addition, shorter surgical times may be related to patient comfort factors mentioned in the previous section.

\section{Increased Accuracy}

If MR imaging is not, or cannot be, optimized for a particular deep brain target, the surgeon must rely on ventricular landmarks, prior knowledge based on established atlases, and MER to identify the appropriate location for electrode implantation. Such indirect imaging methods have led to the initial track being used in $70 \%$ of cases and an average of 2.3 tracts per implanted electrode. , $^{8,37}$ In comparison, a single brain penetration is sufficient in $87-95 \%$ of patients undergoing MRI-guided and MRI-verified DBS; with only one additional track was required in the remainder. ${ }^{37,38}$

During DBS surgery under general anesthesia, it has been theorized that the 
positive-pressure ventilation increases intracranial pressure, which to some extent reduces brain shift. In addition with meticulous entry planning on a gyrus and shorter surgical times are felt to reduce egress of cerebrospinal fluid and pneumocephalus, which further reduces stereotactic inaccuracy from brain shift. ${ }^{39}$ In our practice, we feel that we further reduce these deleterious factors by keeping the patient supine and by drilling only a $2 \mathrm{~mm}$ twist drill hole.

In published series of "asleep" DBS to date, the mean deviation of the implanted electrode when compared to the intended trajectory is only $1.2 \mathrm{~mm} .{ }^{14,15,40}$ While advocates of MER may argue that this low deviation supports precision and not necessarily accuracy, current experience has also shown that the site of best MER activity does not necessarily correlate with best clinical response during intraoperative testing or long-term outcome. ${ }^{41,42}$ Furthermore, in the setting of improved perioperative imaging, there exists no evidence that MER help prevent suboptimal electrode placement. ${ }^{10,43-46}$ In fact, cases of mistaking the recordings of the red nucleus for STN highlight the false sense of security that MER can provide. ${ }^{47}$

\section{Reduced Risk of Intracranial Hemorrhage}

One of the main arguments for DBS to be performed without MER is that there is an increased risk of intracranial hemorrhage with increased brain penetrations. ${ }^{31,48}$ In a 2011 meta-analysis of 109 studies comprising 6,237 patients and 9,890 trajectories to deep nuclei, the estimated per-trajectory intracerebral hemorrhage (ICH) rate was $1.57 \%$ with an estimated mortality rate per trajectory was of $0.14 \%$. The use of MER and multiple trajectories to deep nuclei were both positive predictors of increased ICH risk. ${ }^{49,50}$ As illustrated above, the image-guided image-verified electrode implantation is associated with fewer brain penetrations, which contributes to reducing the associated risk of $\mathrm{ICH}$. When evaluated separately, the overall incidence of $\mathrm{ICH}$ in functional neurosurgery has been reported to be $5.0 \%$, with asymptomatic hemorrhage in $1.9 \%$ of patients, symptomatic hemorrhage in $2.1 \%$ and hemorrhage resulting in permanent deficit or death in $1.1 \%$. In comparison, in 214 patients undergoing image-guided DBS without MER, Zrinzo et al reported a total incidence of $\mathrm{ICH}$ of was $0.9 \%$, with asymptomatic in $0.5 \%$ of patients, symptomatic hemorrhage in $0.5 \%$, and hemorrhage resulting in permanent deficit in $0.0 \%$ of patients. ${ }^{50}$ Overall this equates to a four to five fold increase in hemorrhage risk in awake surgery performed with MER.

\section{CONCLUSION}

Despite the significant differences between these two techniques outlined above, their clinical outcomes and procedural costs to date have been largely the same. While the benefits of neurophysiologic and clinical confirmation advocate for "awake" DBS, the advantages of greater patient comfort, decreased operating time, increased accuracy, and reduced hemorrhagic complications support "asleep" DBS. As such, it is important to discuss these factors with patients considering DBS surgery. Each approach has its own advantages and disadvantages that must be weighed with patient-specific factors, concerns, and preferences.

\section{REFERENCES}

1. Starr PA, Vitek JL, Bakay RA. Ablative surgery and deep brain stimulation for Parkinson's disease. Neurosurgery. 1998;43(5):989-1013; discussion 1013-1015

2. Sironi VA. Origin and evolution of deep brain stimulation. Front Integr Neurosci. 2011;5:42. doi:10.3389/fnint.2011.00042

3. WETZEL N, SNIDER RS. Neurophysiological correlates in human stereotaxis. Q Bull Northwest Univ Med Sch. 1958;32(4):386-392.

4. Beckmann EC. CT scanning the early days. Br J Radiol. 2006;79(937):5-8. doi:10.1259/ bjr/29444122.

5. Britain's brains produce first NMR scans. New Sci. November 1978:588.

6. Summary Of Safety And Effectiveness Data For A Supplemental Premarket Approval Application.; 2002.

7. Products and Procedures for Medtronic Deep Brain Stimulation. http://professional. medtronic.com/pt/neuro/dbs-md/prod/\#. VwHLRhMrLvs. Accessed June 20, 2004.

8. Bronte-Stewart H. Surgical placement of deep brain stimulation leads for the treatment of movement disorders: intraoperative aspects: physiological mapping, test stimulation, and patient evaluation. In: Marks WJ, ed. Deep Brain Stimulation Management. Cambridge, UK: Cambridge University Press: 2015:20-32
9 Zeiler FA Wilkinson M, Krcek JP. Subthalamic nucleus deep brain stimulation: an invaluable role for MER. Can J Neurol Sci. 2013;40(4):572-575.

10. McClelland S. A cost analysis of intraoperative microelectrode recording during subthalamic stimulation for Parkinson's disease. Mov Disord. 2011;26(8):1422-1427. doi:10.1002/ mds.23787.

11. Chabardes S, Isnard S, Castrioto A, et al. Surgical implantation of STN-DBS leads using intraoperative MRI guidance: technique, accuracy, and clinical benefit at 1-year follow-up. Acta Neurochir (Wien). 2015;157(4):729-737.

12. Follett KA, Weaver FM, Stern M, et al. Pallidal versus subthalamic deep-brain stimulation for Parkinson\&apos;s disease. N Engl J Med. 2010;362(22):2077-2091. doi:10.1056/ NEJMoa0907083.

13. Ostrem JL, Ziman N, Galifianakis NB, et al. Clinical outcomes using ClearPoint interventional MRI for deep brain stimulation lead placement in Parkinson's disease. J Neurosurg. 2015:1-9. doi:10.3171/2015.4.JNS15173.

14. Burchiel KJ, McCartney S, Lee A, Raslan AM Accuracy of deep brain stimulation electrode placement using intraoperative computed tomography without microelectrode recording. J Neurosurg. 2013;119(2):301-306

15. Mirzadeh Z, Chapple K, Lambert M, et al. Parkinson's disease outcomes after intraoperative CT-guided "asleep" deep brain stimulation in the globus pallidus internus. J Neurosurg. 2015:1-6 doi:10.3171/2015.4.JNS1550.

16. Mirzadeh Z, Chapple K, Lambert M, Dhall $\mathrm{R}$, Ponce FA. Validation of CT-MRI fusion for intraoperative assessment of stereotactic accuracy in DBS surgery. Mov Disord. 2014;29(14):1788-1795. doi:10.1002/ mds.26056.

17. Mirzadeh Z, Chapple K, Lambert M, et al. Parkinson's disease outcomes after intraoperative CT-guided "asleep" deep brain stimulation in the globus pallidus internus. J Neurosurg. 2016;124(4):902-907. doi:10.3171/2015.4.JNS1550.

18. Sharma M, Deogaonkar M. Accuracy and safety of targeting using intraoperative "O-arm" during placement of deep brain stimulation electrodes without electrophysiological recordings. J Clin Neurosci. 2016 doi:10.1016/j.jocn.2015.06.036.

19. Zrinzo L, Hariz M, Hyam JA, Foltynie T, Limousin P. Letter to the Editor: A paradigm shift toward MRI-guided and MRI-verified DBS surgery. J Neurosurg. February 2016:1-2. doi:10.3171/2015.9.JNS152061.

20. Williams A, Gill S, Varma T, et al. Deep brain stimulation plus best medical therapy versus best medical therapy alone for advanced Parkinson's disease (PD SURG trial): a randomised, open-label trial. Lancet Neurol. 2010;9(6):581-591. doi:10.1016/S14744422(10)70093-4. 
21. Deep-Brain Stimulation of the Subthalamic Nucleus or the Pars Interna of the Globus Pallidus in Parkinson's Disease - NEJM http://www.nejm.org/doi/full/10.1056/ NEJMoa000827. Accessed March 27, 2016

22. Okun MS, Fernandez HH, Wu SS, et al. Cognition and mood in Parkinson's disease in subthalamic nucleus versus globus pallidus interna deep brain stimulation: the COMPARE trial. Ann Neurol. 2009;65(5):586-595. doi:10.1002/ana.21596.

23. Rodriguez-Oroz MC, Moro E, Krack P. Long-term outcomes of surgical therapies for Parkinson's disease. Mov Disord. 2012:27(14):1718-1728. doi:10.1002/ mds. 25214

24. Maldonado IL, Roujeau T, Cif L, et al. Magnetic resonance-based deep brain stimulation technique: a series of 478 consecutive implanted electrodes with no perioperative intracerebral hemorrhage. Neurosurgery. 2009;65(6 Suppl):196 201; discussion 201-202. doi:10.1227/01. NEU.0000342404.14347.FB.

25. Cif L, Vasques X, Gonzalez V, et al. Long-term follow-up of DYT1 dystonia patients treated by deep brain stimulation: an open-label study. Mov Disord. 2010:25(3):289-299. doi:10.1002/mds.22802.

26. Plaha P, Ben-Shlomo Y, Patel NK, Gill SS. Stimulation of the caudal zona incerta is superior to stimulation of the subthalamic nucleus in improving contralateral parkinsonism. Brain. 2006;129(Pt 7):1732-1747. doi:10.1093/brain/awl127.

27. Ostrem JL, Ziman N, Galifianakis NB, et al. Clinical outcomes using ClearPoint interventional MRI for deep brain stimulation lead placement in Parkinson\{ltextquoteright\}s disease. J Neurosurg. October 2015:1-9.

28. Aviles-Olmos I, Kefalopoulou Z, Tripoliti $\mathrm{E}$, et al. Long-term outcome of subthalamic nucleus deep brain stimulation for Parkinson\&apos;s disease using an MRI-guided and MRI-verified approach J Neurol Neurosurg \&amp; Psychiatry. 2014;85(12):1419-1425. doi:10.1136/jnnp2013-306907.

29. Saleh S, Swanson KI, Lake WB, Sillay KA Awake Neurophysiologically Guided versus Asleep MRI-Guided STN DBS for Parkinson Disease: A Comparison of Outcomes Using Levodopa Equivalents. Stereotact Funct Neurosurg. 2015;93(6):419-426. doi:10.1159/000442425.

30. Jacob RL, Geddes J, McCartney S, Burchiel KJ. Cost analysis of awake versus asleep deep brain stimulation: a single academic health center experience. J Neurosurg. November 2015:1-7.

31. Binder DK, Rau GM, Starr PA. Risk factors for hemorrhage during microelectrodeguided deep brain stimulator implantation for movement disorders. Neurosurgery. 2005;56(4):722-732; discussion 722-732
32. Starr PA, Christine CW, Theodosopoulos $P V$, et al. Implantation of deep brain stimulators into the subthalamic nucleus: technical approach and magnetic resonance imaging-verified lead locations. J Neurosurg. 2002;97(2):370-387. doi:10.3171/jns.2002.97.2.0370.

33. Montgomery Jr. EB. Validation of CT-MRI fusion for intraoperative assessment of stereotactic accuracy in DBS surgery. Mov Disord. 2015;30(3):439. doi:10.1002/ mds. 26178 .

34. Harries AM, Kausar J, Roberts SAG, et al. Deep brain stimulation of the subthalamic nucleus for advanced Parkinson disease using general anesthesia: long-term results. J Neurosurg. 2012;116(1):107-113. doi:10.3171/2011.7.JNS11319.

35. Hertel F Züchner M. Weimar I, et al. Implantation of electrodes for deep brain stimulation of the subthalamic nucleus in advanced Parkinson's disease with the aid of intraoperative microrecording under general anesthesia. Neurosurgery. 2006;59(5):E1138; discussion E1138. doi:10.1227/01 NEU.0000245603.77075.55.

36. Maltête $D$, Navarro $S$, Welter $M-L$, et al. Subthalamic stimulation in Parkinson disease: with or without anesthesia? Arch Neurol. 2004;61(3):390-392. doi:10.1001/archneur 61.3 .390

37. Zrinzo L, Foltynie T, Limousin P, Hariz M. Image-verified deep brain stimulation reduces risk and cost with no apparent impact on efficacy. Mov Disord. 2012;27(12):1585 1586; author reply 1586-1587. doi:10.1002/ mds. 25100

38. Starr PA, Martin AJ, Ostrem JL, Talke P. Levesque N, Larson PS. Subthalamic nucleus deep brain stimulator placement using high-field interventional magnetic resonance imaging and a skull-mounted aiming device: technique and application accuracy. J Neurosurg. 2010;112(3):479-490. doi:10.3171/2009.6.JNS081161.

39. Petersen EA, Holl EM, Martinez-Torres I, et al. Minimizing brain shift in stereotactic functional neurosurgery. Neurosurgery. 2010;67(3 Suppl Operative):ons213-ons221; discussion ons221. doi:10.1227/01. NEU.0000380991.23444.08.

40. Chen T, Mirzadeh Z, Chapple K, Lambert M, Dhall R, Ponce FA. "Asleep" deep brain stimulation for essential tremor. J Neurosurg 2015:1-8. doi:10.3171/2015.6.JNS15526.

41. Wodarg F, Herzog J, Reese R, et al. Stimulation site within the MRI-defined STN predicts postoperative motor outcome. Mov Disord. 2012:27(7):874-879. doi:10.1002/ mds. 25006
42. Bour LJ, Contarino MF, Foncke EMJ, et al. Long-term experience with intraoperative microrecording during DBS neurosurgery in STN and GPi. Acta Neurochir (Wien). 2010;152(12):2069-2077. doi:10.1007/s00701010-0835-y.

43. Foltynie T, Zrinzo L, Martinez-Torres I, et al. MRI-guided STN DBS in Parkinson's disease without microelectrode recording efficacy and safety. J Neurol Neurosurg Psychiatry. 2011;82(4):358-363. doi:10.1136/ jnnp.2010.205542.

44. McClelland S, Kim B, Winfield LM, et al. Microelectrode recording-determined subthalamic nucleus length not predictive of stimulation-induced side effects. Neurosurg Focus. 2005;19(5):E13.

45. Ellis T-M, Foote KD, Fernandez $\mathrm{HH}$, et al. Reoperation for suboptimal outcomes after deep brain stimulation surgery. Neurosurgery. 2008:63(4):754-760; discussion 760-761. doi:10.1227/01.NEU.0000325492.58799.35.

46. Hariz MI, Fodstad H. Do microelectrode techniques increase accuracy or decrease risks in pallidotomy and deep brain stimulation? A critical review of the literature. Stereotact Funct Neurosurg. 1999;72(2-4):157-169. doi:29720.

47. Biddell KM, Duker AP, Mandybur G. Can Microelectrode Recording be Used to Accurately Differentiate the Red Nucleus from Subthalamic Nucleus? In: 19th Annual Scientific Meeting of the North American Neuromodulation Society. Las Vegas, NV; 2015.

48. Hariz MI. Safety and Risk of Microelectrode Recording in Surgery for Movement Disorders. Stereotact Funct Neurosurg. 2002;78(3-4):146-157. doi:10.1159/000068960

49. Kimmelman J, Duckworth K, Ramsay T, Voss T, Ravina B, Emborg ME. Risk of surgical delivery to deep nuclei: a meta-analysis. Mov Disord. 2011;26(8):1415-1421. doi:10.1002/ mds. 23770 .

50. Zrinzo L, Foltynie T, Limousin P, Hariz MI. Reducing hemorrhagic complications in functional neurosurgery: a large case series and systematic literature review. J Neurosurg. 2012;116(1):84-94. doi:10.3171/2011.8.JNS101407. 